\title{
Analyse didaktischer Veränderungen durch Digitalisierung. Die Mär von mehr Partizipation?
}

\section{Christina Gloerfeld}

\section{Zusammenfassung}

Dieser Artikel ist ein Beitrag zur Diskussion über die Transformation von Lehr-/Lernprozessen durch Digitalisierung aus didaktischer Perspektive und nimmt dabei gezielt den Bereich der Partizipation in den Blick. Es wird eine empirische Untersuchung der didaktischen Veränderungen durch Digitalisierung an der FernUniversität (FeU) in Hagen vorgestellt. Die Grundlage bildet eine systematische Erfassung der Lehr-/Lernprozesse anhand eines entwickelten analytischen Rahmenmodells, das aus sechs allgemeindidaktischen Modellen abgeleitet ist. Die exemplarischen Ergebnisse werden vor dem Hintergrund aktueller Erkenntnisse diskutiert und geben auf diese Weise Impulse und Reflexionsansätze gegen teilweise stark überzogene Erwartungshaltungen der Veränderungen im Lehren und Lernen. Es ist evident, dass durch Digitalisierung Handlungsräume erweitert werden und Möglichkeiten für neue Handlungsformen und -praxen entstehen. Die Realisierung dieser Potenziale, zum Beispiel im Bereich der Partizipation, befindet sich jedoch noch am Anfang und hat grundlegende Strukturen bisher wenig aufgebrochen.

\section{Schlüsselwörter}

Digitalisierung • Partizipation • Didaktische Veränderungen • Analytisches Rahmenmodell • Lehr-/Lernprozesse • Transformation • Allgemeine Didaktik • Didaktische Modelle

C. Gloerfeld ( $\square)$

Universität Bremen, Bremen, Deutschland

E-Mail: christina.gloerfeld@vw.uni-bremen.de 


\section{$1 \quad$ Einleitung}

Es herrscht weitgehend die Ansicht vor, dass die Digitalisierung im Bildungsbereich angekommen ist. Diese Ansicht beruht darauf, dass durch die Durchdringung des Alltags mit digitalen Medien Digitalisierung zwangsläufig, entsprechend einer Push-Strategie, von den Studierenden in die Hochschulen gedrängt wurde (Dittler und Kreidl 2018; Kerres und Stratmann 2005; Selwyn 2010; Steffens et al. 2018). Gleichzeitig begannen die Universitäten aktiv die Potenziale digitaler Bildungstechnologien, wie Flexibilität (unter anderen Arnold et al. 2018), erweiterte Kommunikations- und Interaktionsmöglichkeiten (unter anderen Knaus 2018) oder die Nutzung von Daten für schnellere oder automatisierte Rückmeldungen an Studierende (Pauschenwein und Lyon 2018) fruchtbar zu machen (Pull-Strategie). Durch die Potenziale der vernetzten Bildungstechnologien wird der Handlungsspielraum in Lehr-/Lernprozessen nicht nur ins Digitale verlängert, sondern es entstehen vielfältige neue Gestaltungsmöglichkeiten, bei denen die Lernenden aktiver ihre Lernprozesse selbst bestimmen oder zur Partizipation angeregt werden können (Arnold et al. 2018). Während also das Veränderungspotenzial durch Digitalisierung unbestritten scheint, besteht Uneinigkeit über das Ausmaß und die Tiefe der Veränderungen.

Dieser Beitrag nimmt die Auswirkungen von Digitalisierung auf Lehr-/ Lernprozesse genauer in den Blick, indem zum einen ein Werkzeug zur differenzierten Erfassung vorgestellt wird und zum anderen Veränderungen bezüglich der Partizipation an Lehr-/Lernprozessen analysiert werden sowie eine Einschätzung der proklamierten Transformation von Lehren und Lernen erfolgt.

\section{Bedingungen und Chancen für Partizipation durch Digitalisierung}

Mit dem Aufkommen digitaler Medien, vor allem aber durch die Entwicklung zum Web 2.0, wurden passive Konsument*innen medialer Inhalte $\mathrm{zu}$ aktiven Prosument*innen, die digitale Angebote mitgestalten und produzieren. Auch in der pädagogischen Diskussion wurden die neuen Möglichkeiten des sogenannten Mitmachnetzes zur Teilhabe, Zusammenarbeit und Interaktion aufgegriffen (Mayrberger 2012). Eine Beteiligung an Entscheidungen und Entscheidungsprozessen, zum Beispiel zur Gestaltung von Angeboten und Interaktionen, wird von Mayrberger als Partizipation bezeichnet (ebenda). Die Möglichkeiten, Lernende aktiv einzubeziehen, finden besondere Resonanz, da das Web 2.0 den populären Ansätzen konstruktivistischer Lerntheorien folgt. Es sind diese Grundlagen 
konstruierten Wissens, kollaborativer Lernprozesse und der Einsatz spielerischer Elemente, die das Web 2.0 für das Lehren und Lernen so attraktiv machen und das Potenzial haben, bisherige Hierarchien zwischen Lehrenden und Lernenden aufzubrechen (Selwyn 2010). Denn eine Einbeziehung der Lernenden in die Gestaltung von Lehr-/Lernprozessen bedingt eine neue Aufteilung von Entscheidungsräumen und Einteilung von Entscheidungsmacht. „Insgesamt lässt sich sagen, dass Partizipation von der Bereitschaft der einen Seite lebt, Verantwortung für Entscheidungen bzw. Entscheidungsmacht abzugeben [sic] und durch die Bereitschaft und Kompetenz der anderen Seite, Verantwortung für Entscheidungen zu übernehmen“ (Mayrberger 2012, S. 4). Partizipation ist ein grundlegend demokratisches Prinzip, das besondere Anforderungen an die Kompetenzen aller Beteiligten stellt (ebenda). Die Befähigung zur Teilhabe an der Gesellschaft ist eine hochschulische Aufgabe, der die Institutionen durch die Erfüllung des Bildungsauftrags nachkommen (sollten) (Hafer et al. 2019). Durch Beteiligung an der Hochschule, in der Kursgestaltung und im Lehr-/Lernprozess können diese übergeordneten Ziele wie Autonomie, Selbstorganisation und Mündigkeit adressiert werden.

Der Begriff Partizipation wird auf unterschiedlichen Ebenen und in verschiedenen Kontexten angewendet. Wannemacher et al. (2019) identifizieren drei Kategorien für die verschiedenen Arten von Teilhabe in einer Untersuchung diverser Zukunfts- und Trendstudien. Die erste Kategorie ist Bildungsteilhabe durch ein diversifiziertes Bildungsangebot, Weiterbildung, berufsbegleitende Bildungsangebote und lebenslanges Lernen. Die zweite Kategorie fasst Bildungsteilhabe durch neue Gestaltungsmöglichkeiten von Lehr-/Lernszenarien zusammen. Mit dem Einsatz digitaler Technologien entstehen didaktische Gestaltungsspielräume, um Lehr-/Lernprozesse unter anderem personalisiert, interaktiv, kollaborativ/kooperativ und/oder selbstbestimmt zu konzipieren. In der dritten Kategorie werden die Aspekte zu Bildungsteilhabe und Bildungsgrenzen zusammengefasst (ebenda).

In diesem Beitrag geht es um Partizipation der zweiten Kategorie auf der Ebene von Lehr-/Lernprozessen. Grundlegende Ideen zur Teilhabe reichen bis in die Reformpädagogik zurück und haben durch die Bologna-Reform 1999 den Wechsel vom Lehren zum Lernen erneut angestoßen (Wildt 2005). Ebenso sind die Ziele der Selbstbestimmung, der Fähigkeit zur kritischen Auseinandersetzung und Teilhabe an der Gesellschaft bereits ab Mitte des 20. Jahrhunderts in den didaktischen Ansätzen von Reich, Schulz oder Winkel wiederzufinden (Mayrberger 2012, 2019). Die neuen Technologien des Web 2.0 brachten einen weiteren Aufschwung für partizipative Ansätze, da sie die didaktischen Anforderungen nach Beteiligung und Eigentätigkeit besser bedienen können. Ullrich et al. lassen sich sogar so weit zusammenfassen, dass Konstruktivismus die angeborene 
Pädagogik des Web 2.0 sein könnte (Ullrich et al. 2008). Die Weiterentwicklung digitaler Medien (Hard- und Software) verstärkt demnach die Ideen und Ansätze zur Partizipation und damit auch den Wandel vom Lehren zum Lernen. Sie ermöglichen eine aktive Mitgestaltung digitaler Inhalte, die zunehmend einfacher, schneller sowie günstiger (ebenda) und damit für nahezu jeden realisierbar wird (Knaus 2018). Damit stehen Möglichkeiten bereit, die Kontrolle über den Lernprozess, die Entscheidungen zu Auswahl und Gestaltung von Lernen auf Lernende zu übertragen (Downes 2005) und damit Partizipation zu realisieren. Aktuell wird diese Verschiebung von Verantwortung vor allem unter den Begriffen des Selbstorganisierten oder Selbstgesteuerten Lernens diskutiert und insbesondere im Zugriff auf Open Educational Resources (OER), Personal Learning Environments (PLE) und im Einsatz von Learning Analytics (LA), durch Visualisierungen eigener Lernprozesse und -fortschritte, aber auch im Vergleich mit anderen deutlich (Arnold et al. 2018; Getto et al. 2018; Kerres 2018).

Zur Beschreibung und Beurteilung von Partizipation stellt Mayrberger ein Stufenmodell mit vier Partizipationstypen und neun Partizipationsformen vor (2019, S. 98 f.): Typ 1 ist die Nicht-Partizipation mit den Formen Dekoration/Anweisung und Fremdbestimmung oder Instrumentalisierung. Ein Beispiel sind klassisch instruktionale Designs, wie die ersten Lernmaschinen. Typ 2 mit den Formen Einbeziehung, Teilhabe und Alibi-Teilnahme wird als Vorstufe der Partizipation, Pseudo- oder Scheinbeteiligung bezeichnet. In Typ 3 handelt es sich um die eigentliche Partizipation als Selbstbestimmung, Mitbestimmung und Mitwirkung. Bei der Form der Selbstbestimmung sind die Lernenden eigeninitiativ, werden aber von Lehrenden begleitet, unterstützt, angeleitet. Mitbestimmung lässt ihnen eine Teilhabe an Entscheidungen. Davon abzugrenzen ist die Mitwirkung, bei der die Entscheidungsmacht bei den Lehrenden verbleibt, die Lernenden jedoch Impulse und Ideen einbringen können. Beispiele für diesen Typ sind begleitet erstellte User-Generated-Contents, die im Sinne von OER mit anderen Lernenden geteilt werden, Ansätze des forschenden Lernens, der Einsatz der Inverted-Classroom-Methode oder schlicht die Berïcksichtigung der Erwartungen oder Rückmeldungen der Studierenden (Mayrberger 2019). An der Hochschule Fresenius setzen die Studierenden beispielsweise selbstbestimmt ein Praxisprojekt als Teil des Curriculums um, bei dem sie von Mentor*innen unterstützt werden (Faber und Hood 2019). Mitbestimmung findet dort im Rahmen der Seminare statt, indem die Studierenden Themen vorschlagen, erarbeiten und vortragen können, die sich aus Sicht der Lehrenden in den Kontext des Seminars integrieren lassen (ebenda). Typ 4 bezeichnet Autonomie, die über Partizipation hinausgeht und die Selbstverwaltung und Selbstorganisation meint. Beispiele sind Makerspaces und studentisch selbstorganisierte Lerngruppen oder Lerntools sowie 
offene Bildungsinitiativen (ebenda). Diese werden zwar von Studierenden initiiert und umgesetzt, allerdings liegt es an den Hochschulen, Rahmenbedingungen zu schaffen, die das Entstehen solcher Initiativen fördern und eine nachhaltige Implementation ermöglichen (Sporer et al. 2011). An der TU Berlin entwickelten Studierende eigeninitiativ und selbstständig das Seminar Blue-Engineering, das nun im Regelbetrieb auch an anderen Hochschulen eingesetzt wird (Baier und Neef 2019).

Es bleibt also genau zu betrachten, welche Potenziale und Erwartungen Digitalisierung in Lehr-/Lernprozessen erfüllt und welche mit besonderem Blick auf Partizipation realisiert werden.

\section{Veränderungen von Lehr-/Lernprozessen durch Digitalisierung am Beispiel der FeU}

\subsection{Design und Werkzeug der empirischen Untersuchung}

Dazu wird nachfolgend exemplarisch eine empirische Untersuchung didaktischer Veränderungen durch Digitalisierung an der FernUniversität (FeU) in Hagen vorgestellt (Gloerfeld 2020), in der insbesondere die Auswirkungen auf den Bereich der Partizipation beschrieben und vor dem Hintergrund des aktuellen Forschungsstands diskutiert werden. Im Untersuchungszeitraum Juli bis November 2016 wurden leitfadengestützte Expert*inneninterviews (Gläser und Laudel 2009; Meuser und Nagel 2005, 2013) mit 17 Lehrenden und Lernenden durchgeführt und im Sinne des maximal kontrastierenden Vergleichs (Meuser und Nagel 2013) hinsichtlich Statusgruppe, Geschlecht, Tätigkeitszeitraum und Fakultätszugehörigkeit mit der inhaltlich strukturierenden qualitativen Inhaltsanalyse nach Mayring (2008, 2016) ausgewertet. Da die Rolle des Leitmediums, das die digitale Epoche terminiert ${ }^{1}$, an der FernUniversität in Hagen Moodle zugesprochen werden konnte, beziehen sich die Interviews als Folge dieser Prämisse auf Lehr-/Lernprozesse vor und nach der Einführung der Lernplattform. Die Grundlage der Datenerhebung und -auswertung ist ein analytisches Rahmenmodell des Lehr-/Lernprozesses, welches seine grundlegende Struktur in den einzelnen Bestandteilen abbildet.

\footnotetext{
${ }^{1}$ Neue Formen der Gesellschaft lassen sich anhand der in ihnen dominanten Medien bezeichnen und in Epochen einteilen (Baecker 2016). Diese bilden eine fruchtbare Grundlage für die Analyse und Identifikation von Veränderungen durch Digitalisierung im Bildungsbereich, indem ein Vergleich zwischen der medialen Epoche des Digitalen mit der davorliegenden stattfindet (Pietraß 2017; Schwalbe und Meyer 2010).
} 
Dieses Werkzeug zur Analyse didaktischer Prozesse wurde systematisch entlang der semantischen und dimensionalen Analyse nach Kromrey ${ }^{2}$ (2009) aus sechs allgemeindidaktischen Modellen abgeleitet. Die Auswahl der Modelle folgte dem Anspruch, die Bandbreite allgemeindidaktischer Theorien abzubilden. Daher wurden die am meisten verbreiteten Modelle für jede wissenschaftstheoretische Position nach Jank und Meyer (2009) und ein Modell zu jedem Leitbegriff nach der Einteilung von Kron (2008) ausgewählt. Tab. 1 zeigt das Ergebnis in der Kreuztabelle aus den wissenschaftstheoretischen Positionen, dialektisch, hermeneutisch, konstruktivistisch, empirisch-analytisch (Jank und Meyer 2009) und den Leitbegriffen Bildung, Lernen, Interaktion, System, Konstruktion (Kron 2008): das Perspektivschema zur Unterrichtsplanung (Klafki 1994), das Berliner Modell nach Heimann, Otto und Schulz (Heimann 1962; Schulz 1976), das Modell kybernetischer Unterrichtsplanung (von Cube 1982), das Analyse- und Planungskonzept der kritisch-kommunikativen Didaktik (Winkel 1999), das dialektische Modell von Unterricht (Klingberg 1984a) und die Mindestperspektiven auf die Planungsreflexion (Reich 2012).

In der Betrachtung und dem Vergleich der Bestandteile der Modelle werden nur wenige Überschneidungen direkt ersichtlich. Partizipation als Bestandteil kommt bei Reich (2012) unter dem Begriff „Partizipatives Lehren und Lernen“ und bei Winkel (1999) als „Mitverantwortung und Mitgestaltung“ sehr offensichtlich vor. In der tieferen Betrachtung der Bedeutung der Begriffe und Bestandteile werden weitere partizipative Momente bei Klafki (1994) unter „Erweisbarkeit und Überprüfbarkeit“ oder bei Heimann et al. (Heimann 1962; Schulz 1976) in den ,,anthropologischen/anthropologisch-psychologischen Voraussetzungen und Folgen“, als Mitsteuerung des Unterrichtsprozesses durch die Lernenden, deutlich. Auf dieser Ebene konnten entlang der semantischen Analyse zunächst die Bedeutung und die Zusammenhänge der Bestandteile der einzelnen Modelle auf begrifflicher Ebene herausgearbeitet werden. Diese Gesamtheit der Bedeutungen der Begriffe bildete die Grundlage für die dimensionale Analyse, in der in mehreren Arbeitsschritten die Begriffe in 460 Beschreibungsdimensionen, Teildimensionen und Merkmalsausprägungen differenziert wurden, um empirisch anschlussfähig zu sein. Auf dieser Ebene konnten im Folgenden Überschneidungen und Unterschiede für die Selektion der grundlegenden Dimensionen des Lehr-/Lernprozesses nutzbar gemacht werden und ihre Zusammenfassung erfolgen. Demnach wurden Dimensionen, die Gleiches in den Modellen beschreiben,

\footnotetext{
${ }^{2}$ Das Vorgehen der semantischen und dimensionalen Analyse basiert auf dem Ansatz von Kromrey (2009, S. 113 ff.) zur empirischen Übersetzung des Forschungsproblems, indem es theoretische Begriffe mit der empirischen Wirklichkeit verbindet.
} 


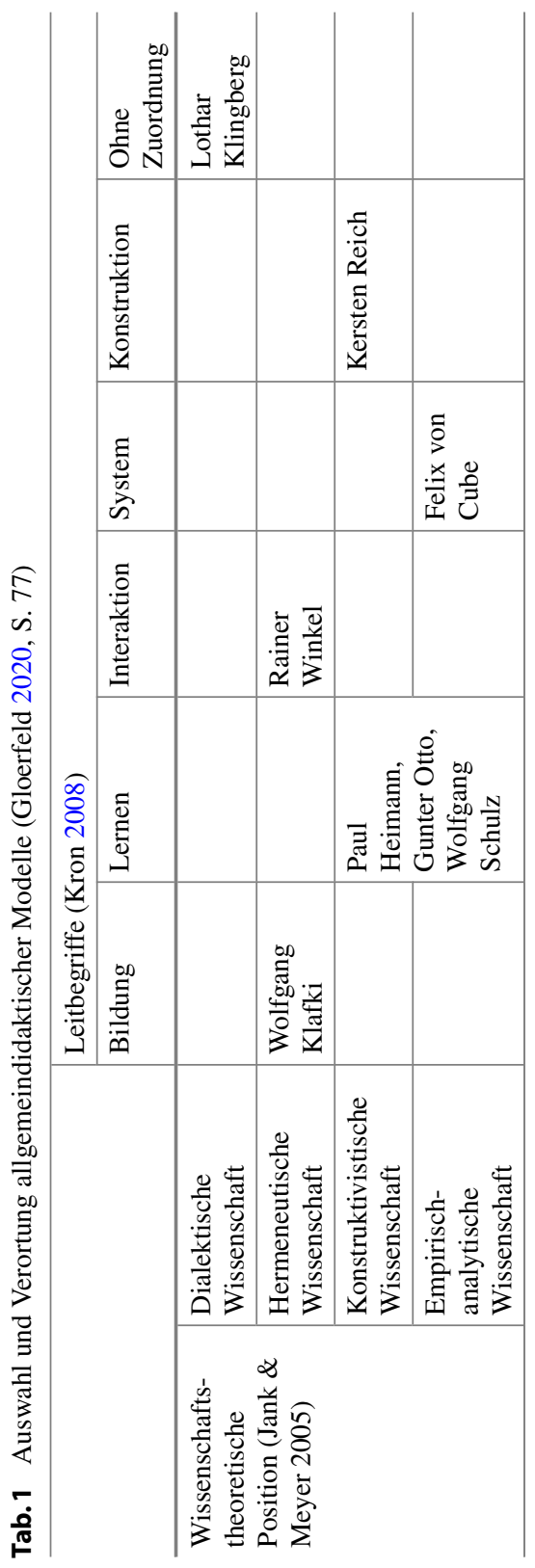


beispielsweise Partizipation und Beteiligung, als Gruppe zusammengefasst. Für die Beschreibung von Partizipation und Beteiligung ergaben sich so die beiden Dimensionen Orte/Ansatzpunkte für Partizipation und Beteiligung und Maß an Mitgestaltung der Lernenden. Über die Teil-Dimensionen der Orte/Ansatzpunkte, Unterrichtsprozess, -planung, Inhalte, Methoden, Medien und Ziele, wird die Berücksichtigung der verschiedenen Modelle deutlich. Der Raum für spontane Unterrichtsgestaltung bei von Cube (1982) oder die Mitsteuerung der Lehrenden und Lernenden über ihre Bereitschaft und Aktivitäten bei Heimann et al. (Heimann 1962; Schulz 1976) finden sich zum Beispiel im Ansatzpunkt Unterrichtsprozesse. Die „Ebenen der Partizipation“ (Reich 2012) oder das „Maß an Mitgestaltung" (Winkel 1999) sind in der Teil-Dimension Maß an Mitgestaltung zusammengefasst. Tab. 2 zeigt in der linken Spalte Beschreibungsdimensionen der Begriffe der Modelle und in der rechten Spalte die zusammengefassten Dimensionen über alle Modelle hinweg am Beispiel von Partizipation und Beteiligung. Im Gegensatz zur Einteilung nach Partizipationstypen nach Mayrberger (2019), die sich auf den Grad der Beteiligung beziehen, wurden in der Untersuchung zusätzlich Ansatzpunkte für Partizipation berücksichtigt.

Insgesamt konnten auf diese Weise elf Gruppen als Bestandteile für ein analytisches Rahmenmodell des Lehr-/Lernprozesses gebildet werden: Lehrende, Lernende, Inhalt, Methode, Medien, Ziel, Kontext(-Bedingungen), Beziehungen, Bewertungen und Kontrolle, Partizipation und Beteiligung sowie Störungen.

Die Bestandteile und ihre Bestimmung über Dimensionen und TeilDimensionen bildeten die theoretische Grundlage für die empirische Untersuchung. Zum einen lieferten sie die Themenbereiche für die Leitfragen der Interviewleitfäden und zum anderen ein deduktives Kategoriensystem für die inhaltlich-strukturierende qualitative Inhaltsanalyse nach Mayring (2008), das im Verlauf der Datenauswertung mit induktiven Kategorien ergänzt wurde.

\subsection{Darstellung und Interpretation der Ergebnisse}

Nachfolgend wird nur ein knapper Einblick in einige Ergebnisse der umfassenden Untersuchung aller elf Bestandteile (Gloerfeld 2020) gegeben, aber eine fokussierte Wiedergabe der Kategorie Partizipation und Beteiligung vorgenommen.

Insgesamt fällt in einem zunächst quantitativen Überblick der inhaltsanalytischen Auswertung auf, dass es fast doppelt so viele Kodierungen (1.306) für den Zeitraum nach der Einführung von Moodle (nM) gibt wie für den Zeitraum davor (vM) (725). Bemerkenswert ist, dass die Verteilung auf die elf Kategorien nahezu identisch ist. Dabei fallen insgesamt in die drei häufigsten Kategorien Medien 
Tab. 2 Selektion und Zusammenfassung der Beschreibungsdimensionen aus den Modellen am Beispiel von Partizipation. (Eigene Darstellung)

\begin{tabular}{|c|c|}
\hline $\begin{array}{l}\text { Abgeleitete Beschreibungsdimensionen der Begriffe } \\
\text { in den Modellen (Bsp. Partizipation) }\end{array}$ & $\begin{array}{c}\text { Selektierte und zusammengefasste Dimensionen } \\
\text { Partizipation und Beteiligung }\end{array}$ \\
\hline \multicolumn{2}{|l|}{$\begin{array}{l}\text { Heimann et al., Berliner Modell (Heimann 1962; } \\
\text { Schulz 1976) }\end{array}$} \\
\hline - Mitsteuerung des Unterrichtsprozesses & $\begin{array}{l}\text { - Orte/Ansatzpunkte für Partizipation und } \\
\text { Beteiligung }\end{array}$ \\
\hline o Aktivitäten und Bereitschaft & o Unterrichtsplanung \\
\hline o der Lernenden & o Unterrichtsprozess \\
\hline o der Lehrenden & o Aktivitäten und Bereitschaft der Beteiligung \\
\hline $\begin{array}{l}\text { von Cube, Kybernetische Unterrichtsplanung (1982, } \\
\text { S. } 350 \text { ) }\end{array}$ & o der Lernenden \\
\hline $\begin{array}{l}\text { - Entscheidungsspielraum in der Unterrichtssituation } \\
\text { (Umfang und Art) }\end{array}$ & o der Lehrenden \\
\hline o für Lehrende & o Erhebung/Beobachtung des Unterrichts \\
\hline o Beteiligung der Lernenden & o Reflexion der Planung und Konsequenzen \\
\hline $\begin{array}{l}\text { Winkel, Analyse und Planungskonzept der kritisch- } \\
\text { kommunikativen Didaktik (1999, S. 99) }\end{array}$ & o Evaluation/Bewertung \\
\hline - Maß an Mitgestaltung der Lernenden & o Inhalt \\
\hline stellvertretende Entscheidung, & o Methoden \\
\hline behutsame Partizipation, & o Medien \\
\hline regressiv-komplementäres Agieren, & o Ziele \\
\hline$\cdot$ symmetrisches Agieren & - Maß an Mitgestaltung der Lernenden \\
\hline $\begin{array}{l}\text { Reich, Mindestperspektiven auf die } \\
\text { Planungsreflexion (2012, S. 251) }\end{array}$ & $\begin{array}{l}\cdot \text { von Informieren und Begründen bis zu } \\
\text { Selbstbestimmung }\end{array}$ \\
\hline $\begin{array}{l}\text { - Ebenen (Grade) der Partizipation im Lehren und } \\
\text { Lernen }\end{array}$ & \\
\hline $\begin{array}{l}\cdot \text { Notwendigkeiten von Unterrichtsplänen } \\
\text { (-methoden) transparent machen/ } \\
\text { Unterrichtsentscheidungen begründen, erklären }\end{array}$ & \\
\hline gemeinsame Unterrichtsplanung & \\
\hline $\begin{array}{l}\text { Erhebungen/Beobachtungen, bspw. Reflecting } \\
\text { Teams }\end{array}$ & \\
\hline$\cdot$ Reflexion der Planung und Konsequenzen & \\
\hline$\cdot$ Evaluation/Bewertung & \\
\hline - Beteiligung der Lernenden bei den Inhalten & \\
\hline o Selbstverantwortung & \\
\hline o Auswahl, Erarbeitung der Inhalte & \\
\hline o Intentionen & \\
\hline - Art der Methoden- und Medienwahl & \\
\hline$\cdot$ Selbstständig durch die Lernenden & \\
\hline$\cdot$ Unterstützt durch die Lehrenden & \\
\hline $\begin{array}{l}\text { Klafki, Perspektivschema zur Unterrichtsplanung } \\
\text { (1994) }\end{array}$ & \\
\hline $\begin{array}{l}\text { Begriff im Modell: Erweisbarkeit/Überprüfbarkeit } \\
\text { - Gestaltung des Bewertungsprozesses }\end{array}$ & \\
\hline $\begin{array}{ll}\quad \text { Beteiligung der Lernenden bei Festlegung der } \\
\text { Kriterien }\end{array}$ & \\
\hline $\begin{array}{l}\text { Kriterien } \\
\text { o Selbstbewertungsmöglichkeit der Lernenden }\end{array}$ & \\
\hline Legende: $\quad-\quad$ Dimension (Merkmal) & \\
\hline
\end{tabular}


(26-28\%), Kontext (16-18\%) und Methode (14-15\%) fast $60 \%$ aller Kodierungen. Eine strukturelle Veränderung, beispielsweise durch eine Verschiebung von Schwerpunkten der Kategorien, ist nicht erkennbar.

Im Gegensatz dazu zeigen sich innerhalb der einzelnen Bestandteile des Lehr-/ Lernprozesses sehr wohl Veränderungen. Es gibt ein wachsendes Medien- und Informationsangebot sowie erweiterte Zugriffs- und Kommunikationsmöglichkeiten, welche die Komplexität von Lehren und Lernen steigern.

In der Kategorie Partizipation und Beteiligung verändern sich durch die Einführung der Lernumgebung zunächst die Möglichkeiten der Aktivität und Beteiligung im Unterrichtsprozess, da neben den Präsenzveranstaltungen noch Foren in Moodle hinzukommen und auch E-Mails häufiger genutzt werden. Die meisten Beteiligungsmöglichkeiten zeigen sich in der Bewertung und Kontrolle, hier ist vor und nach der Einführung die Wahl von Prüfungsthemen oder die eigene Ausgestaltung eines Prüfungsthemas möglich. Während die Themenwahl bei Seminarund Abschlussarbeiten vor der Einführung von Moodle teilweise sehr frei ist, wird nach der Einführung von Moodle diese Freiheit erst in späteren Semestern und nicht in der Studieneingangsphase gewährt. Bezüglich der Inhalte gibt es vM eine strukturierte Abfrage zu den Studienmaterialien, welche die Studierenden zur Bewertung und Rückmeldung postalisch zugesendet bekommen. Mit Moodle gibt es diesen Prozess nicht mehr, allerdings stehen in den Studiengängen mehr Wahlmodule zur Verfügung und die Studierenden nutzen die Foren der Plattform für (auch ungefragte) Rückmeldungen. Vereinzelt beziehen die Lehrenden die Studierenden zum gemeinsamen Erarbeiten von Inhalten mit kollaborativen Medien ein. Neu hinzugekommen ist im Bereich Medien, dass die Studierenden eigeninitiativ Software oder Applikationen vorschlagen, die zum Lehren und Lernen genutzt werden können. Die Möglichkeiten, sich in die inhaltliche, methodische oder mediale Gestaltung von Lehr-/Lernprozessen einzubringen, haben sich demnach erweitert. Im Vergleich war die Flexibilität im Bereich der Bewertung und Kontrolle der Zeit vor Moodle teilweise allerdings größer. Durch die ergänzenden Lernangebote, Informationen und Kommunikationskanäle verändert sich auch das $\mathrm{Maß}$ an Mitgestaltung. Entscheidungsräume erweitern sich und Entscheidungen werden transparenter und teilweise deutlicher begründet, beispielsweise durch Wahlmodule, Studieninformationen und Modulhandbücher. Allerdings obliegt den Lernenden an der FernUniversität schon immer ein besonderes Maß an Selbstbestimmung, da sie ihr Studium selbst organisieren, Studienzentren aufsuchen und Lerngruppen bilden, wozu die FernUniversität die Organisationsstruktur mit Räumlichkeiten und Unterstützungsmöglichkeiten durch Tutor*innen vor Ort anbietet. 


\section{Diskussion}

Die hier vorgestellten Ergebnisse stammen aus einer qualitativen Untersuchung der FernUniversität von 2016, die es vor diesem Hintergrund zu reflektieren gilt. Das heißt, dass erst in der Diskussion mit aktuellen Erkenntnissen die Ergebnisse eingeschätzt und allgemeine Entwicklungstendenzen aufgezeigt werden können. Die besondere Betonung des Medieneinsatzes und der Kommunikationsmöglichkeiten sind eine Besonderheit des Fernstudiums, das beides zur Bedingung für Lehren und Lernen hat. Gleichzeitig bilden beide Aspekte relevante Schwerpunkte, wenn es um die Potenziale digitaler Medien für die Hochschulbildung insgesamt geht (Arnold et al. 2018; Knaus 2018).

Eine gesteigerte Verbreiterung des Lehr-/Lernangebots durch die Offenheit von Lernressourcen und die gesteigerten Zugriffsmöglichkeiten zeigen sich im gesamten Hochschulbereich (Arnold et al. 2018; Schön et al. 2017). Ebenso werden erweiterte Kommunikations- und Interaktionsmöglichkeiten für digitale Lehr-/Lernszenarien als Potenzial ausgelobt (BMBF 2016; Schön et al. 2017). Die damit entstandenen Räume für Beteiligung in Unterrichtsprozessen bieten vor allem vor-partizipative Möglichkeiten der Einbeziehung oder AlibiTeilnahme des Typs 2 nach Mayrberger (2019). Betrachtet man die Umsetzung von Partizipation in Bezug zu den didaktischen Modellen, wird vor allem die Begründung und Transparenz von Unterrichtsplänen und -entscheidungen nach Reich (2012) realisiert, während ansonsten maßgebliche Ansprüche der konstruktivistischen Didaktik mit höheren Graden an Partizipation, der Methoden oder Medienwahl kaum stattfinden. Ebenso werden aus dem Blickwinkel der kritischkommunikativen Didaktik von Winkel (1999) hauptsächlich die ersten beiden Stufen von Partizipation stellvertretende Entscheidung und behutsame Partizipation erreicht und kaum regressiv-komplementäres oder symmetrisches Agieren von Lehrenden und Lernenden. Allerdings entstehen mehr Möglichkeiten für Aktivitäten im Unterrichtsprozess im Sinne des Berliner Modells (Heimann 1962; Schulz 1976) oder des Entscheidungsspielraums in der Unterrichtssituation der kybernetischen Didaktik (von Cube 1982). Die Realisierung von Partizipation nach Typ 3 findet sich über die Berücksichtigung von Rückmeldungen (Mitwirkung) und Wahlfreiheiten bei Themen und Prüfungsformen (Mitbestimmung und Selbstbestimmung) nur teilweise an der FernUniversität. Allgemein besteht das Problem, dass interaktive und interdisziplinär angelegte Szenarien aufwendiger in der Vorbereitung und Betreuung sind, denn je selbstbestimmter der Lernprozess ist, desto größer wird der Anspruch an die Betreuung und Begleitung durch die Lehrenden (Kauffeld et al. 2019). Über die Organisationsstruktur des Fernstudiums mit orts- und zeitunabhängigen Studienmöglichkeiten, einer technologischen 
Infrastruktur zur Vernetzung sowie dem Netz aus Studienzentren vor Ort schafft die FernUniversität bereits grundlegende Voraussetzungen für Partizipation. Hier zeigt sich nach Sporer et al. eine positive Entwicklung ,zu aktiver Teilhabe und stärkerer Öffnung gegenüber neuen Ideen und Problemlösungen ,von unten“ motiviert" (2011, S. 232). Allerdings bilden partizipative Lehr-/Lernprozesse insgesamt noch eine zunehmende Herausforderung für die Hochschulen (Mayrberger 2012).

Eine aktuelle Untersuchung der Veränderungen an der FernUniversität wäre sicherlich aufschlussreich, in diesem Beitrag zeigt die Untersuchung für sich genommen vor allem die Einsatzmöglichkeiten des analytischen Rahmenmodells für eine differenzierte Untersuchung der Veränderungen von Lehr-/Lernprozessen.

\section{$5 \quad$ Fazit und Ausblick}

Das Ziel dieses Artikels war, eine empirisch gestützte Einschätzung der Transformation didaktischer Prozesse durch Digitalisierung mit dem Fokus auf Partizipation zu liefern und ein Werkzeug für eine differenzierte Analyse zur formalen Beschreibung von Lehr-/Lernprozessen vorzustellen. Dazu wurden die Ergebnisse einer empirischen Untersuchung didaktischer Veränderungen durch Digitalisierung wiedergegeben und diskutiert. Der Beitrag schließt auf diesen zwei Ebenen an die Fachdiskussion zur Digitalisierung in der Hochschulbildung an. Erstens stellt er mit dem analytischen Rahmenmodell ein Werkzeug für eine differenzierte Analyse vor, um Lehr-/Lernprozesse formal zu beschreiben und konkrete Aussagen zu deren Veränderungen machen zu können. Wie oben am Beispiel von Partizipation ausgeführt, liefern die differenzierten Kategorien eine fruchtbare Struktur. Über die Betrachtung von Partizipation auf zwei Ebenen, der Ansatzpunkte und des Ausmaßes, sind die Ergebnisse über die Typen von Partizipation nach Mayrberger (2019) in der Beurteilung anschlussfähig und zeigen konkrete Ansatzpunkte für didaktische Maßnahmen zur Förderung von Partizipation auf. In dieser Hinsicht weist der Beitrag, zweitens, eine inhaltliche Anschlussfähigkeit auf, indem die Ergebnisse der Untersuchung Argumente zur Bewertung einer Transformation durch Digitalisierung mit besonderem Fokus auf Partizipation bereitstellen.

Insgesamt bleiben die aktive Einbindung der Lernenden und das tatsächliche Teilen von Entscheidungsräumen erst einmal eine Option, die mit den Errungenschaften der Digitalisierung einfacher und vielfältiger wahrzunehmen ist. Allerdings zeigen sich derartig konstruktivistische Umsetzungen erst in einzelnen multimodalen Lernangeboten. Es werden vor allem Vorstufen von Partizipation 
gedacht, in denen es um Mitgestaltungsmöglichkeiten im Unterrichtsprozess geht, mehr Informationen bereitgestellt oder Rückmeldungen zur Lehre eingeholt werden. Entlang der beiden Unterkategorien von Partizipation und Beteiligung aus dem Rahmenmodell werden andere Ansatzpunkte aufgezeigt, die zum Beispiel in der Unterrichtsplanung ansetzen und auch die Entscheidungen über Inhalte, Medien, Methoden und Bewertungen als partizipative Elemente in den Vordergrund rücken. Auf diese Weise könnten konstruktivistische Szenarien umgesetzt und die Erfüllung des Bildungsauftrags gezielter angegangen werden.

Digitalisierung eröffnet also Handlungsräume und schafft Gestaltungsmöglichkeiten für Partizipation, die jedoch ohne Anreize laut Handke (2017) weder von den Lehrenden noch den Lernenden wahrgenommen werden. Es bestätigt sich, dass didaktische Veränderungen nicht technologisch induziert werden und einfach passieren, sondern dass es gestaltungsbereite, -willige und -fähige Pädagog*innen braucht, um neue Einsatzszenarien $\mathrm{zu}$ entwickeln und umzusetzen (Arnold et al. 2018; Mayrberger 2012; Riplinger und Schiefner-Rohs 2017). Demnach setzt mit Digitalisierung nicht automatisch eine Veränderung ein, sondern es werden vielmehr bestehende Praktiken in digitalem Gewand ausgeführt. Dies stellt keine Transformation dar, denn erst durch das Entstehen neuer Anwendungen und Handlungsformen könnte von Transformation gesprochen werden (Allert 2018; Baecker 2007; Pietraß 2017).

Mit den Ergebnissen wird eine relativierende Position zu den überzogenen Erwartungen an Digitalisierung eingenommen und es werden kritisch die Diskrepanz zwischen proklamierter und realisierter Partizipation an Lehr-/Lernprozessen sowie Ansatzpunkte für Mitgestaltung im konstruktivistischen Sinne aufgezeigt und Anregungen für anschließende Diskussionen gegeben.

\section{Literatur}

Allert, H. (2018). Kritische Bestandsaufnahme: Bildungsverständnis und Digitalisierung. Synergie. Fachmagazin für Digitalisierung in der Lehre, 6, 16-19.

Arnold, P., Kilian, L., Thillosen, A. M., \& Zimmer, G. M. (2018). Handbuch E-Learning: Lehren und Lernen mit digitalen Medien (5. Aufl.). Bielefeld: W. Bertelsmann.

Baecker, D. (2007). Studien zur nächsten Gesellschaft. Frankfurt a. M.: Suhrkamp.

Baecker, D. (2016). Wie verändert Digitalisierung unser Denken und unseren Umgang mit der Welt? In B. Leukert \& R. Gläß (Hrsg.), Handel 4.0: Die Digitalisierung des Handels - Strategien, Technologien, Transformation (S. 3-24). Berlin: Springer Gabler.

Baier, A., \& Neef, M. (2019). Studierende lehren und lernen sozial-ökologische Verantwortung. Die Neue Hochschule. DNH für anwendungsbezogene Wissenschaft und Kunst, 61(5), 8-11. 
Bundesministerium für Bildung und Forschung (BMBF). (2016). Bildungsoffensive für die digitale Wissensgesellschaft. Strategie des Bundesministeriums für Bildung und Forschung. Berlin: BMBF.

Dittler, U., \& Kreidl, C. (2018). Entwicklung des Hochschulwesens und dessen aktuelle Situation in der kritischen Betrachtung. In U. Dittler \& C. Kreidl (Hrsg.), Hochschule der Zukunft: Beiträge zur zukunftsorientierten Gestaltung von Hochschulen (S. 15-34). Wiesbaden: Springer VS.

Downes, S. (2005). E-Learning 2.0. eLearn magazine, 10 (1). https://elearnmag.acm.org/fea tured.cfm?aid=1104968. Zugegriffen: 30. Juni 2020.

Faber, O., \& Hood, A. G. (2019). Den Augenblick ergreifen: Eine neue Lernperspektive außerhalb des Curriculums. Die Neue Hochschule. DNH für anwendungsbezogene Wissenschaft und Kunst, 61(5), 16-19.

Getto, B., Hintze, P., \& Kerres, M. (2018). (Wie) Kann Digitalisierung zur Hochschulentwicklung beitragen? In B. Getto, P. Hintze, \& M. Kerres (Hrsg.), Digitalisierung und Hochschulentwicklung: Proceedings zur 26. Tagung der Gesellschaft für Medien in der Wissenschaft e. V., Medien in der Wissenschaft, 74 (S. 13-26). Münster: Waxmann.

Gläser, J., \& Laudel, G. (2009). Experteninterviews und qualitative Inhaltsanalyse als Instrumente rekonstruierender Untersuchungen (3 (überarb). Wiesbaden: Springer VS.

Gloerfeld, C. (2020). Auswirkungen von Digitalisierung auf Lehr- und Lernprozesse. Didaktische Veränderungen am Beispiel der FernUniversität in Hagen. Wiesbaden: Springer VS.

Hafer, J., Mauch, M., \& Schumann, M. (2019). Teilhabe in einer digitalen Bildungswelt. In J. Hafer, M. Mauch \& M. Schumann (Hrsg.), Teilhabe in der digitalen Bildungswelt. Medien in der Wissenschaft, 75. (S. 9-13). Münster: Waxmann.

Handke, J. (2017). Handbuch Hochschullehre Digital: Leitfaden für eine moderne und mediengerechte Lehre (2 überarbeitete). Baden-Baden: Tectum.

Heimann, P. (1962). Didaktik als Theorie und Lehre. In K. Reich \& H. Thomas (Hrsg.), (1976) Didaktik als Unterrichtswissenschaft (1. Aufl., S. 142-167). Stuttgart: Klett.

Jank, W., \& Meyer, H. (1991). Didaktische Modelle: Alle Schulformen (5. Aufl.). Berlin: Cornelsen.

Kauffeld, S., Herrmann, C., Heuer, K., Pulst, S., \& Kühne, M. (2019). GLuE - Gemeinsames Lernen und Erfahren. Eine innovative und interdisziplinäre Lehr-Lern-Kooperation. In S. Robra-Bissantz, O. J. Bott, N. Kleinefeld, K. Neu \& K. Zickwolf (Hrsg.), Teaching Trends 2018. Die Präsenzhochschule und die digitale Transformation (S. 36-42). Münster: Waxmann.

Kerres, M. (2018). Bildung in der digitalen Welt: Wir haben die Wahl. denk-doch-mal.de. Das online-Magazin für Arbeit-Bildung-Gesellschaft, (Berufliche) Bildung fürs Leben, 02-18. https://learninglab.uni-due.de/sites/default/files/Kerres_denk-doch-mal.pdf. Zugegriffen: 20. Okt. 2020

Kerres, M., \& Stratmann, J. (2005). Bildungstechnologische Wellen und nachhaltige Innovation: Zur Entwicklung von E-Learning an Hochschulen in Deutschland. In M. Kerres \& R. Keil-Slawik (Hrsg.), Hochschulen im digitalen Zeitalter: Innovationspotenziale und Strukturwandel; Education Quality Forum 2004, 2 (S. 29-47). Münster: Waxmann.

Klafki, W. (1994). Neue Studien zur Bildungstheorie und Didaktik. Zeitgemäße Allgemeinbildung und kritisch-konstruktive Didaktik (4, durchges. Aufl.). Weinheim u. a.: Beltz. 
Klingberg, L. (1984). Einführung in die Allgemeine Didaktik. Vorlesungen (6. Aufl.). Berlin: Volk und Wissen. Volkseigener Verlag.

Knaus, T. (2018). [Me]nsch - Werkzeug - [I]nteraktion. Theoretisch-konzeptionelle Analysen zur „Digitalen Bildung“ und zur Bedeutung der Medienpädagogik in der nächsten Gesellschaft. MedienPädagogik: Zeitschrift für Theorie und Praxis der Medienbildung, „Digitale Bildung “. Medienbezogene Bildungskonzepte für die „nächste Gesellschaft“, 31 (S. 1-35). https://doi.org/10.21240/mpaed/31/2018.03.26.x.

Kromrey, H. (2009). Empirische Sozialforschung: Modelle und Methoden der standardisierten Datenerhebung und Datenauswertung (12., überarb. und erg). Stuttgart: Lucius \& Lucius.

Kron, F. W. (2008). Grundwissen Didaktik (5 überarb). München: Reinhardt.

Mayrberger, K. (2012). Partizipatives Lernen mit dem Social Web gestalten. Zum Widerspruch einer verordneten Partizipation. MedienPädagogik: Zeitschrift für Theorie und Praxis der Medienbildung, Partizipationschancen, (S. 1-25). https://doi.org/10.21240/mpaed/21/ 2012.01.12.X.

Mayrberger, K. (2019). Partizipative Mediendidaktik Gestaltung der (Hochschul-)Bildung unter den Bedingungen der Digitalisierung. Weinheim: Beltz.

Mayring, P. (2008). Qualitative Inhaltsanalyse: Grundlagen und Techniken. Weinheim: Beltz.

Mayring, P. (2016). Einführung in die qualitative Sozialforschung: Eine Anleitung zu qualitativem Denken (6., überarbeitete). Weinheim: Beltz.

Meuser, M., \& Nagel, U. (2005). ExpertInneninterviews - vielfach erprobt, wenig bedacht. Ein Beitrag zur qualitativen Methodendiskussion. In A. Bogner, B. Littig \& W. Menz (Hrsg.), Das Experteninterview: Theorie, Methode, Anwendung (2. Aufl., S. 71-94). Wiesbaden: Springer VS.

Meuser, M., \& Nagel, U. (2013). Experteninterviews: Wissenssoziologische Voraussetzungen und methodische Durchführung. In B. Friebertshäuser \& A. Prengel (Hrsg.), Handbuch qualitative Forschungsmethoden in der Erziehungswissenschaft, (4., durchgesehene Aufl.) (S. 457-472). Weinheim: Juventa.

Pauschenwein, J., \& Lyon, G. (2018). Ist die Zukunft der Hochschullehre digital? In U. Dittler \& C. Kreidl (Hrsg.), Hochschule der Zukunft: Beiträge zur zukunftsorientierten Gestaltung von Hochschulen (S. 145-165). Wiesbaden: Springer VS.

Pietraß, M. (2017). Was ist das Neue an ,digitaler Bildung“? Zum hochschuldidaktischen Potenzial der elektronischen Medien. Erziehungswissenschaft, 28(55), 19-27.

Reich, K. (2012). Konstruktivistische Didaktik: Das Lehr- und Studienbuch mit OnlineMethodenpool (5., erw). Weinheim: Beltz.

Riplinger, T., \& Schiefner-Rohs, M. (2017). Medieneinsatz in der Hochschullehre. Akademische Lehr-Lernkonzepte zwischen Zumutung und Zu-Mutung. https://doi.org/10.13154/ rub.105.94.

Schön, S., Ebner, M., Schön, M., \& Haas, M. (2017). Digitalisierung ist konsequent eingesetzt ein pädagogischer Mehrwert für das Studium: Thesen zur Verschmelzung von analogem und digitalem Lernen auf der Grundlage von neun Fallstudien. In C. Igel (Hrsg.), Bildungsräume: Proceedings der 25. Jahrestagung der Gesellschaft für Medien in der Wissenschaft, 72 (S. 11-19). Münster, New York: Waxmann.

Schulz, W. (1976). Unterricht-Analyse und Planung. In P. Heimann, G. Otto \& W. Schulz (Hrsg.), Unterricht: Analyse und Planung (8., unveränd. Aufl.) (S. 13-47). Hannover: Schroedel. 
Schwalbe, C., \& Meyer, T. (2010). Umbauten im und am Bildungsraum. Zum medieninduzierten Wandel der Kommunikationsstrukturen in der Hochschulbildung. In P. Grell, W. Marotzki, \& H. Schelhowe (Hrsg.), Neue digitale Kultur- und Bildungsräume (S. 27-50). Wiesbaden: Springer VS.

Selwyn, N. (2010). A new education for a new digital age? Towards a critical analysis of young people, education and the contemporary digital landscape. In P. Grell, W. Marotzki, \& H. Schelhowe (Hrsg.), Neue digitale Kultur- und Bildungsräume (S. 13-25). Wiesbaden: Springer VS.

Sporer, T., Dürnberger, H., \& Hofhues, S. (2011). Lernen durch aktive Mitgestaltung? Herausforderungen offener Bildungsinitiativen im Umfeld von Hochschulen. In H. Dürnberger, S. Hofhues, \& T. Sporer (Hrsg.), Offene Bildungsinitiativen: Fallbeispiele, Erfahrungen und Zukunftsszenarien (S. 229-232). Münster: Waxmann.

Steffens, Y., Schmitt, I. L., \& Aßmann, S. (2018). Mediennutzung Studierender: Über den Umgang mit Medien in hochschulischen Kontexten. Systematisches Review nationaler und internationaler Studien zur Mediennutzung Studierender. https://doi.org/10.13154/ rub.106.95.

Ullrich, C., Borau, K., Luo, H., Tan, X., Shen, L., \& Shen, R. (2008). Why Web 2.0 is Good for Learning and for Research: Principles and Prototypes. Proceedings of the 17th International Conference on World Wide Web, April 21-25, 2008, Beijing, China. New York: ACM.

von Cube, F. (1982). Kybernetische Grundlagen des Lernens und Lehrens (4., neubearb. Aufl.). Stuttgart: Klett-Cotta.

Wannemacher, K., Lübcke, M., \& Seyfeli, F. (2019). Things to Come. Digitalisierung und Bildungsteilhabe. Eine Trendanalyse zur Hochschulbildung der Zukunft. In J. Hafer, M. Mauch \& M. Schumann (Hrsg.), Teilhabe in der digitalen Bildungswelt (S. 78-88). Münster: Waxmann.

Wildt, J. (2005). Vom Lehren zum Lernen-Hochschuldidaktische Konsequenzen aus dem Bologna-Prozess für Lehre, Studium und Prüfung. Kurzfassung eines Vortrags zur Expertentagung des EWFT „From Teaching to Learning“, Berlin. https://www.ewft.de/files/Wildt-05-Vom\%20Lehren\%20zum\%20Lernen-hochschul didaktische\%20Konsequenzen.pdf. Zugegriffen: 20. Okt. 20.

Winkel, R. (1999). Die kritisch-kommunikative Didaktik. In H. Gudjons \& R. Winkel (Hrsg.), Didaktische Theorien (10. Aufl.). Hamburg: Bergmann + Helbig. 
Open Access Dieses Kapitel wird unter der Creative Commons Namensnennung 4.0 International Lizenz (http://creativecommons.org/licenses/by/4.0/deed.de) veröffentlicht, welche die Nutzung, Vervielfältigung, Bearbeitung, Verbreitung und Wiedergabe in jeglichem Medium und Format erlaubt, sofern Sie den/die ursprünglichen Autor(en) und die Quelle ordnungsgemäß nennen, einen Link zur Creative Commons Lizenz beifügen und angeben, ob Änderungen vorgenommen wurden.

Die in diesem Kapitel enthaltenen Bilder und sonstiges Drittmaterial unterliegen ebenfalls der genannten Creative Commons Lizenz, sofern sich aus der Abbildungslegende nichts anderes ergibt. Sofern das betreffende Material nicht unter der genannten Creative Commons Lizenz steht und die betreffende Handlung nicht nach gesetzlichen Vorschriften erlaubt ist, ist für die oben aufgeführten Weiterverwendungen des Materials die Einwilligung des jeweiligen Rechteinhabers einzuholen. 